RACAR : Revue d'art canadienne

Canadian Art Review

RACAR

\title{
Aki Odehi : cicatrices de la Terre-Mère, Centre d'exposition de Val-d'Or, 22 juin au 26 août 2018, commissaire : Sonia Robertson
}

\section{Julie Graff}

Volume 44, numéro 1, 2019

URI : https://id.erudit.org/iderudit/1062158ar

DOI : https://doi.org/10.7202/1062158ar

Aller au sommaire du numéro

\section{Éditeur(s)}

UAAC-AAUC (University Art Association of Canada | Association d'art des universités du Canada)

\section{ISSN}

0315-9906 (imprimé)

1918-4778 (numérique)

Découvrir la revue

Citer ce compte rendu

Graff, J. (2019). Compte rendu de [Aki Odehi : cicatrices de la Terre-Mère, Centre d'exposition de Val-d'Or, 22 juin au 26 août 2018, commissaire : Sonia Robertson]. RACAR : Revue d'art canadienne / Canadian Art Review, 44(1), 107-109. https://doi.org/10.7202/1062158ar

Tous droits réservés (C) UAAC-AAUC (University Art Association of Canada | Association d'art des universités du Canada), 2019
Ce document est protégé par la loi sur le droit d'auteur. L'utilisation des services d'Érudit (y compris la reproduction) est assujettie à sa politique d'utilisation que vous pouvez consulter en ligne.

https://apropos.erudit.org/fr/usagers/politique-dutilisation/ 
conceptualism and the return to pictorialism in The Destroyed Room, with emphasis upon mutual connotations of sexual violation. The argument here is that Wall and Wallace, specifically in the latter's extended photographic installations An Attack on Literature (1975) and Image/Text (1979), were attuned to current feminist debates on representation and engaged with the dynamics of male-female relationships in charged ways effectively overlooked by critics.

Despite the extended contextualizing of Vancouver art and history that will certainly broaden its readership-the emergence of a West Coast school of painting, the careful tracing of visiting artists and other communities' activities as they intersect with the artists under discussion, and the debates within the community in relation to art institutions, especially the shifting role of the Vancouver Art Gallery-the text to this point still feels like a book about Jeff Wall. Indeed, it is an adaptation and expansion of the author's PhD dissertation on Wall. In this extended discussion, his work remains paradigmatic of the central thesis, notwithstanding the attention paid to lan Wallace and Christos Dikeakos.

Shifting gears, the final chapter engages entirely with feminist challenges to this narrative. It sets out a welcome chronicle of diverse activities in dance and performance, filmmaking and experimental video, production spaces such as Women in Focus, Reelfeelings and Womens Inter-Art Society, influential guest speakers, programs at the VAG, community-building festivals and the critical voices of Avis Lang Rosenberg and Sara Diamond, among others. While these developments took place parallel to the activities of the avantgarde under discussion, Modigliani claims male photo-conceptualists assimilated feminist critiques of representation in the 1970s that emerged from psychoanalytic discourse and from theories of the gaze and sexed subjectivities. In other words, this integration of the feminine into the "creative masculine bachelor-subject" involved the "theoretical/feminist" rather than the "maternal/feminine" (202) strain that characterized earlier modernists, fending off unwanted criticism of their avant-garde gambits and assuring institutional and market support.

This assimilation, though, did not include the significantly aligned production of women such as Marian Penner Bancroft whose photographic series, engaged with local Vancouver sites and incorporating text and sculptural aspects, were not unlike the defeatured landscape photos of her male peers. Her use of the medium to negotiate subjective experiences of the urban environment, however, disqualified her from being associated with their conceptualist strategies. Reviews and catalogue essays failed to discuss her work beyond the local and the personal, rendering her work irrelevant to larger artworld concerns. These are the pitfalls that Liz Magor navigated in her attempts to elude perceptions of her work as "romantic" or "sentimental" or being about nature rather than a mediation of the environment through material processes shaped by phenomenological concerns. At the same time, the controversy sparked by her photo series Fieldwork (1989) over the appropriation of Indigenous cultural practices marked a collision between a modernist art discourse that married formal innovation to social progress and the politics of representation, where those left out of the picture claimed their space.

This is a deeply researched and carefully argued account of how avant-garde formations have continued to marginalize women's artistic production even during periods of feminist agitation, though any one of the chapters could be a stand-alone reading at the graduate level. In her conclusion, Modigliani's considers the publication of Vancouver Anthology in 1993, which includes Carol Williams "A Working Chronology of Feminist
Cultural Activities and Events in Vancouver: 1970-1990" and Marcia Crosby's "Construction of the Imaginary Indian," as an intervention into developing regional histories of art. But by this time the critical edge of local photo-conceptualist photographers had been subsumed by an outstanding level of international success commensurate with intense local real estate development and global expansion of art brands compatible with neoliberal values. The book could well have ended there, but Modigliani chose to end with a reading of Jeff Wall's The Giant (1992) as a feminist tableau, a conclusion that confounds feminist analyses of the nude in representation and seems to me needlessly conciliatory. That said, this is an admirable work that brings dispassionate clarity to persistent hegemonic structures in the art world. I

Dr. Christine Conley is an art historian and independent curator with expertise in issues of feminism and gender, the ethics of difference, cultural translation, political violence, and armed conflict.

-cconley@geotext.ca

\section{Aki Odehi: cicatrices de la Terre-Mère}

Centre d'exposition de Val-d'Or

22 juin au 26 août 2018

commissaire: Sonia Robertson

Julie Graff

Durant l'été 2018, l'exposition Aki Odehi: cicatrices de la Terre-Mère présentait au Centre d'exposition de Val-d'Or un projet de performances réalisé l'année précédente dans la région de l'Abitibi-Témiscamingue sous la direction de l'artiste et commissaire innue Sonia Robertson, avec la collaboration du Centre d'amitié autochtone de Val-d'Or. L'exposition Aki Odehi, «lieux de cœur» en langue anicinabek, a été conçue en deux 
temps dès ses prémices. Robertson a d'abord organisé les performances, dans la ville de Val-d'Or et ses environs, en réunissant cinq artistes autochtones et allochtones: les artistes anicinabek Kevin Papatie et Karl Chevrier, l'artiste et poétesse crie Virginia Pésémapéo Bordeleau, et les Québécois d'euro-descendance Véronique Doucet et Jacques Baril. Puis, elle a mis en exposition, de manière engageante et réflexive, des traces des performances et des finalités de dialogue et de conciliation qui avaient guidé leur production.

Cette exposition et les performances dont elle rassemblait les traces s'inscrivaient dans le développement d'un ensemble de projets artistiques performant et problématisant les appels à l'action de la Commission de vérité et réconciliation (CVR, 2008-2015). À la fois célébrée et critiquée, tout particulièrement pour son usage du terme «réconciliation" qui sous-tend une conciliation préalable, la cvr a entrainé plusieurs mouvements sociaux et institutionnels, et a profondément influencé le paysage de la deuxième moitié des années 2010. En effet, dans l'objectif de créer des espaces de sécurité culturelle pour les survivant.e.s venu.e.s témoigner, plusieurs formes d'actions esthétiques ont été organisées lors des témoignages de la CVR. Par ailleurs, des expositions ont aussi été développées comme outil pédagogique, cherchant souvent à éduquer la population allochtone. En parallèle, des commissaires, et particulièrement des commissaires d'art, ont cherché à problématiser le travail de la cvr en remettant en cause ses méthodologies, en confrontant le regard des visiteurs, ou en créant des espaces de dialogue et de négociation ${ }^{1}$. Dans Arts of Engagement: Taking Aesthetic Action In and Beyond the Truth and Reconciliation Commission of Canada, les chercheur.e.s Dylan Robinson et Keavy Martin parlent ainsi d' «action esthétique» pour désigner l'affect

Karl Chevrier, Run \& Hide, 2018. Vue de l'installation dans l'exposition Aki odehi: cicatrices de la TerreMère. Commissaire: Sonia Robertson. Centre d'exposition de Val-d'Or. Photo: Carmelle Adam.

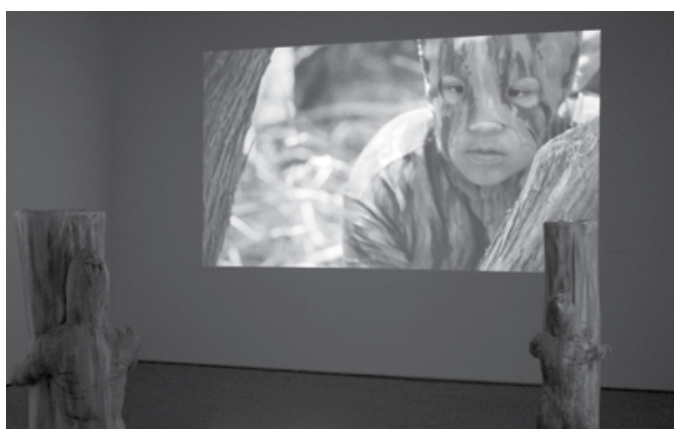

émotif d'un ensemble de stratégies sensibles, d'actions et de créations, mises en place dans le contexte de la CVR, avec leur pouvoir de transformation sociale. ${ }^{2}$

Pensé à la suite de la publication du rapport de la CVR en 2015 et de ses appels à l'action, le projet $A k i$ Odehi a pris une importance supplémentaire à partir de décembre de la même année, quand des allégations de sévices perpétrés par les forces de l'ordreà l'encontre de plusieurs femmes autochtones de Val-d'Or ont été rendues publiques. Si aucune suite n'a été donnée aux plaintes déposées, ${ }^{3}$ celles-ci ont mené à la création d'une commission d'enquête sur les relations entre les Autochtones et certains services publics au Québec.

Aki Odehi avait ainsi à cœur d'offrir des espaces de réparation et de guérison, et d'encourager un renouvellement des relations entre Autochtones et Allochtones d'Abitibi-Témiscamingue. La commissaire Sonia Robertson, qui possède elle-même une vaste expérience en art-thérapie, a donc invité les artistes à réaliser des œuvres in situ, éphémères, inspirées des pratiques en Land Art et en art relationnel, afin de créer des expériences artistiques, esthétiquement et éthiquement fortes, offrant des espaces de dialogue, de conciliation et de réconciliation entre les différentes communautés de la région. Les performances étaient précédées d'une longue période de recherche et de consultation auprès d'aîné.e.s anicinabek, ce qui a permis d'identifier plusieurs lieux importants de rassemblement et de mémoire, considérés comme des «lieux de cœur».

Chaque artiste a ensuite travaillé sur un lieu de cœur résonnant avec sa propre pratique et expérience, et a exploré les blessures marquant ces lieux et les gestes nécessaires à leur cicatrisation. L'exposition poursuivait le travail engagé en 2017, en prolongeant cet espace de dialogue et de sensibilisation. Une première partie de l'exposition rapportait le processus de recherche et de création, alors que photographies, vidéos et textes (rédigés par Sonia Robertson) retraçaient le déroulement de chacune des performances. Par exemple, l'usage de la vidéo par drone permettait de souligner, dans l'espace d'exposition, l'inscription des performances dans le territoire par un jeu d'éloignement visuel. La documentation vidéo évitait par ailleurs le piège de la fictionnalisation des performances en rendant visibles leurs étapes de réalisation et la présence du public. L'exposition présentait non seulement des œuvres et objets utilisés durant les performances, mais aussi des œuvres produites par la suite qui poursuivaient la démarche des artistes. Bien qu'elles n'aient pas été initialement prévues, elles nourrissaient la visite de l'exposition, en montrant le potentiel créatif et innovateur d'une collaboration réussie.

Dans sa performance Run \& Hide, Karl Chevrier revisitait l'histoire des écoles résidentielles, dont il est luimême un survivant. Run \& Hide était un parcours participatif se déroulant 
dans la Forêt Enchantée où les Anicinabek ont vécu, et qui fait maintenant partie du lieu historique national du Fort-Témiscamingue/Obedjiwan. L'artiste était assisté pour ce parcours de quinze membres de sa communauté, toutes générations confondues. Ce chemin a étél'occasion pour les différent.e.s participant.e.s d'échanger, de transmettre et de se réapproprier l'histoire, voire de la réécrire: par exemple, les enfants portaient un camouflage aux couleurs d'écorce pour se cacher des agents des Affaires indiennes, joués par deux participants, venus les arracher à leur famille. Sculpteur de formation, Chevrier a par la suite produit plusieurs sculptures de ces enfants et de leur costume, qu'il a présentées dans l'exposition.

Dans Les Sept Feux, Jacques Baril proposait un rassemblement entre la communauté de Pikogan et la population allochtone de Gallichan, afin d'explorer l'importance de la pointe Apitipik comme lieu d'échange. Faisant participer les deux communautés, il a peint sept toiles, qu'il a ensuite symboliquement immolées par le feu sur le lac, face à la pointe, dans une cérémonie de purification. De cette performance est née une installation des restes carbonisés des toiles, présentée au Centre d'exposition.

Virginia Pésémapéo Bordeleau a produit une œuvre mêlant poésie, performance et Land Art, intitulée Poésie en marche pour Sindy, rendant hommage aux femmes autochtones disparues et assassinées, et plus particulièrement à Sindy Ruperthouse, disparue depuis 2014 et toujours recherchée. Accompagnant les parents et les proches de Ruperthouse, elle a créé des jardins reproduisant les lettres S-I-N-D-Y dans plusieurs lieux emblématiques de Val-d'Or, durant deux marches poétiques auxquelles la population locale a été conviée.

Kevin Papatie est revenu sur le déracinement de sa communauté, dont le territoire ancestral a été en partie inondé pour faire place à une centrale hydroélectrique. Pour son installation 0tipi, il est allé prélever des souches sur ce territoire pour les installer dans le parc de la source Gabriel, à Val-d'Or, et mener à bien une performance revenant sur les événements principaux de sa vie, et invitant le public à réfléchir et à échanger sur les questions du déracinement et de la déculturation. Les souches ont ensuite envahi l'espace de l'exposition, avec une série de documents témoignant du déracinement de la communauté de Papatie.

Finalement, Véronique Doucet a fait appel à la population de l'Abitibi-Témiscamingue pour récupérer et coudre des bas solitaires afin de constituer un immense tipi-jupe, tout en recueillant des témoignages d'abus et d'agressions commis à l'encontre de femmes. Elle a ensuite passé soixante-douze heures nichée dans un arbre, en haut du tipi-jupe qui retombait jusqu'au sol, près du Lac Barrière, afin de transmuter poétiquement la souffrance des femmes. Les témoignages n'ont pas été transposés dans l'exposition ni même documentés, respectant ainsi les confidences recueillies, selon une approche contraire à un extractivisme intellectuel. Toutefois, l'exposition de la tente cousue et des objets utilisés durant la performance permet de reconstituer l'espace créé par Doucet le temps de sa performance.

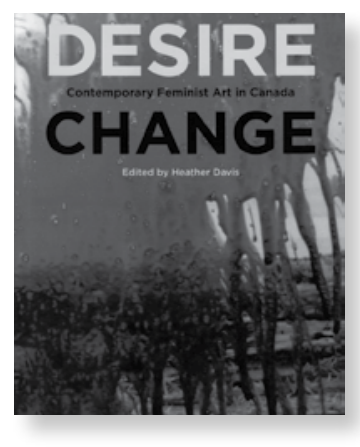

Le projet Aki Odehi de Sonia Robertson a ainsi utilisé l'espace muséal pour créer des espaces de dialogues entre artistes et public, entre différentes communautés, autochtones
Heather Davis, ed.

Desire Change: Contemporary Feminist Art in Canada

Montreal: McGill-Queens University

Press and MAWA, 2017

328 pp. 94 colour illus.

$\$ 45.00$ (cloth) ISBN 9780773549371

et allochtones, des espaces de revendication, d'information, de guérison et action, ancrés dans les histoires l'institution de remplir ses responsabilités sociales, à l'instar d'autres expositions commissariées par des praticien.ne.s autochtones, tel.le.s Sylvie Paré (Oubliées ou Disparues, 20152019) ou encore Guy Sioui Durand (De Tabac et de foin d'odeur. Là où sont nos rêves, 2019). Ces commissaires mettent ainsi au cœur de leur pratique l'engagement sensible et la transformation sociale, pensant de ce fait des solutions dans le contexte actuel de décolonisation des institutions au sein de la société canadienne.

REMERCIEMENT Je remercie Carmelle Adam, directrice du Centre d'exposition de Val-d'Or, ment fournies.

Julie Graff est doctorante à l’Université de Montréal et à l’École des Hautes Études en Sciences Sociales.

ulie.graff@umontreal.ca yond the Truth and Reconciliation Commission of Canada Waterloo, Wilfrid Laurier University Press, 2016.

2. Dylan Robinson et Keavy Martin, «'The

3. Jonathan Montpetit. "What's next for the Indigenous women of Val-d'Or?," CBC News, novembre 2016, https://www.cbc.ca/news/ canada/montreal/val-dor-indigenous-women-1.3858658 (consulté le 30 août 2018).

Natalie Loveless

Angela Davis has often been quoted as saying "I take my identity from my politics, not my politics from my identity." ${ }^{1}$ This would be an apt epigraph to Heather Davis's beautifully 